\title{
Highlights in acute respiratory failure
}

\author{
Raffaele Scala ${ }^{1}$ and Leo Heunks ${ }^{2}$ \\ Affiliations: ${ }^{1}$ Pulmonology and Respiratory Intensive Care Unit, S. Donato Hospital, Arezzo, Italy. ${ }^{2}$ Dept of \\ Intensive Care, VU University Medical Centre, Amsterdam, The Netherlands.
}

Correspondence: Raffaele Scala, Pulmonology and Respiratory Intensive Care Unit, S. Donato Hospital, Via Fonte Veneziana 8, Arezzo, 52100, Italy. E-mail: raffaele_scalaahotmail.com

@ERSpublications

ARF management requires an escalation therapeutic strategy based on application of a wide range of ventilatory and non-ventilatory interventions; there are many unanswered questions that need to be addressed in the near future http://ow.ly/xbPi30iUP2y

Cite this article as: Scala R, Heunks L. Highlights in acute respiratory failure. Eur Respir Rev 2018; 27: 180008 [https://doi.org/10.1183/16000617.0008-2018].

Acute respiratory failure (ARF) is a devastating condition for patients that results from either impaired function of the respiratory muscle pump or from dysfunction of the lung. ARF is a challenging field for clinicians working both within and outside the intensive care unit (ICU) and respiratory high dependency care unit environment because this heterogeneous syndrome is associated with a high hospital morbidity and mortality rate, ethical issues in managing end of life decisions and increased consumption of healthcare resources.

In acute hypercapnic respiratory failure (i.e. pump failure) an imbalance exists between the load imposed on the respiratory muscles and the capacity of the muscle pump [1]. This category mainly includes patients with acute exacerbation of chronic obstructive pulmonary disease (COPD), as well as patients with neuro-miopathies, chest wall deformities and obesity. The in-hospital mortality of patients hospitalised for COPD exacerbation is $2-8 \%$ (up to $15 \%$ for ICU patients), with a 1-year mortality of $22-43 \%$. Readmissions after hospitalisation for an exacerbation are frequent events, ranging from $14 \%$ to $16 \%$ in the first month after discharge and $25-58 \%$ in the first year [2].

Acute hypoxaemic failure covers miscellanea of causes of lung damage including acute cardiogenic pulmonary oedema, pneumonia and trauma. Acute respiratory distress syndrome (ARDS) is a frequent cause of severe hypoxaemia. This clinical syndrome is characterised by acute inflammatory lung injury, associated with increased pulmonary vascular permeability, increased lung weight, and loss of aerated lung tissue [3]. In its most severe form (arterial oxygen tension/inspiratory oxygen fraction $<100 \mathrm{mmHg}$ ), hospital mortality is $\sim 42 \%$ [3]. The insight in the pathophysiology of acute hypoxaemic failure has improved over the past decade. This has important consequences for treatment and monitoring of patients with ARF.

The management of ARF may require an "escalation therapeutic strategy" based on the application of a wide range of ventilatory and non-ventilatory interventions (figure 1) [1,4]. The rationale for applying these artificial supports is essentially to buy time for the aetiological therapy to reverse the cause of the acute decompensation of the respiratory system while avoiding/minimising the potential lung injuring effects of therapeutic interventions, such as ventilator-induced lung injury. In noninvasive ventilation (NIV) a dedicated interface is used, while with invasive mechanical ventilation (IMV) assistance is provided through an endotracheal tube or tracheostomy. New therapeutic options include high-flow nasal cannula [5], noninvasive and invasive cough assist strategies, high-frequency chest wall oscillation, and

Received: Feb 052018 | Accepted: March 052018

Provenance: Commissioned article, peer reviewed.

Copyright OERS 2018. ERR articles are open access and distributed under the terms of the Creative Commons Attribution Non-Commercial Licence 4.0. 


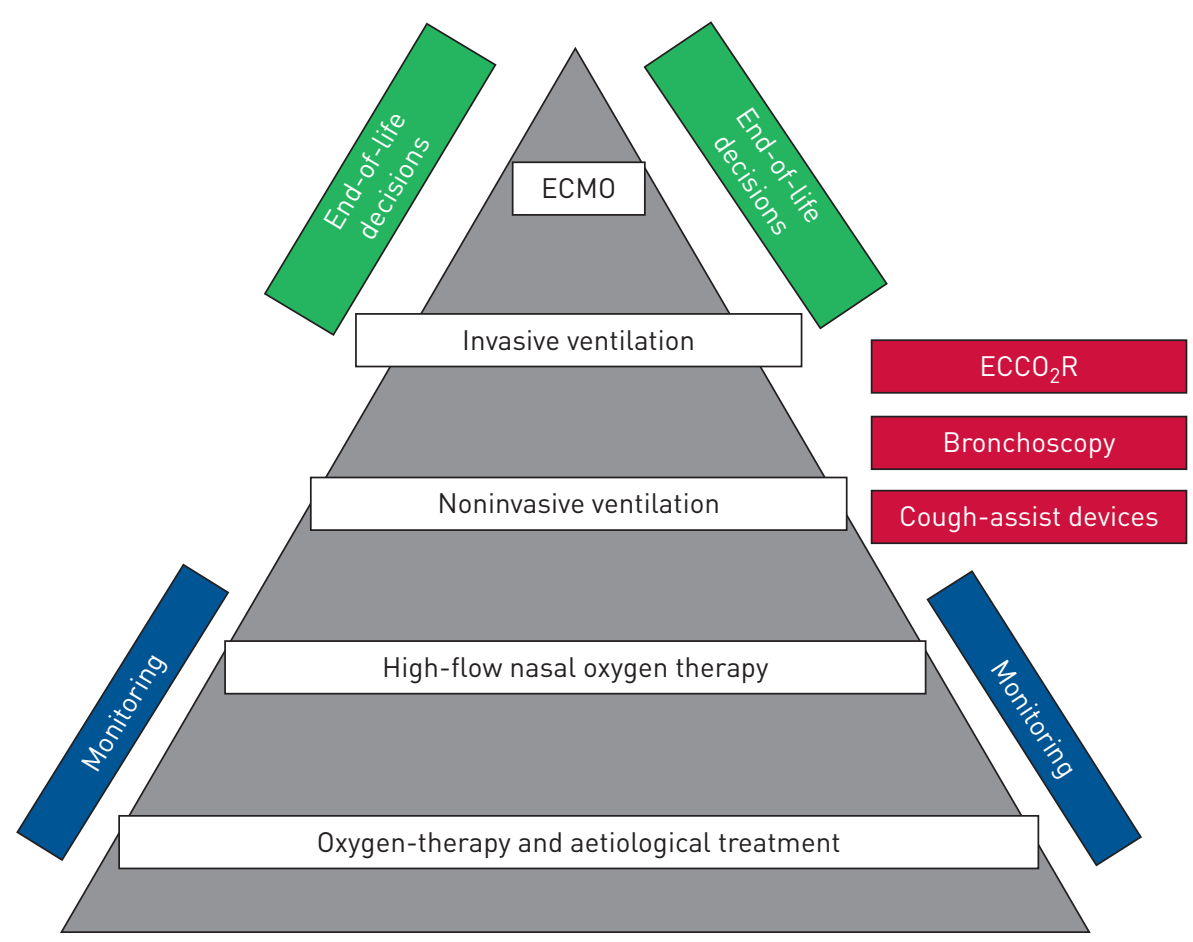

FIGURE 1 Escalation therapeutic strategy, integrative supports and management of acute respiratory failure. ECMO: extracorporeal membrane oxygenation; $\mathrm{ECCO}_{2} \mathrm{R}$ : extracorporeal $\mathrm{CO}_{2}$ removal. Reproduced and modified from [6] with permission.

extracorporeal $\mathrm{CO}_{2}$ removal $\left(\mathrm{ECCO}_{2} \mathrm{R}\right)$ [6]. Finally, refractory hypoxaemia during IMV may require additional integrated strategies such as prone ventilation, neuromuscular blockage, and implementation of "artificial lung" by means of extracorporeal membrane oxygenation [7].

In order to update the reader, the European Respiratory Review is launching a series of invited articles on relevant acute respiratory failure topics (e.g. management of ARDS, monitoring of critically ill patients during NIV, and NIV treatment of ARF), written by experts in the field, both from clinical and scientific perspectives.

In the current issue, Bos et al. [8] focused the reader's interest on the clinical challenges associated with both ventilatory and non-ventilator therapeutic strategies within daily real-life practice and in the research field. This excellent review discusses clinical management and research perspective in patients with ARDS. Therefore, several take-home messages may be easily identified and can be summarised as follows.

1) Changes in the management of ARDS have been highlighted. Thanks to improved insight in the pathophysiology of ventilator-induced lung injury it is now clear that the main goal of mechanical ventilation is not linked to the achievement of the best blood gas and lung recruitment but to prevent and limit the harm induced by ventilatory support [9]. Permissive hypercapnia, permissive hypoxaemia and permissive atelectasis have been introduced as the main target of so-called lung protective ventilation.

2) Bos et al. [8] discuss the best evidenced-based conventional protective targets (i.e. tidal volume $<6 \mathrm{~mL} \cdot \mathrm{kg}^{-1}$ predicted body weight, pressure plateau $<30 \mathrm{cmH}_{2} \mathrm{O}$, driving pressure $<15 \mathrm{cmH}_{2} \mathrm{O}$ ) and alveolar recruitment options (e.g. higher positive end-expiratory pressure (PEEP) levels) in ARDS patients. However, the favourable impact of this protective strategy on clinical outcomes is not warranted in all clinical and physiological ARDS scenarios. Accordingly, the authors stress the concept that some ventilator strategies are likely to work in some subsets of patients while they could be ineffective or even harmful in others.

3) The authors discuss rescue strategies when conventional treatment fails. Although it is questionable whether prone positioning is a rescue strategy, or merely part of routine care for ARDS patients, the role of other interventions, including neuromuscular blockade, $\mathrm{ECCO}_{2} \mathrm{R}$ and extracorporeal membrane oxygenation is less established.

4) The role of NIV in patients with hypoxaemic ARF is a subject of debate [10]. The peculiar advantages of NIV versus IMV (i.e. lower risk of ventilator-associated infections) should be carefully weighed against the 
recently reported potential deleterious effects of the former in patients with moderate-to-severe ARDS [5]. This may result from high tidal volumes in patients with hypoxaemic failure under NIV [11], although this needs further confirmation. Interestingly, there may be a role for high-flow nasal cannula in patients with ARF, as discussed by Bos et al. [8].

5) Despite their rationale in the early dysreactive phase of ARDS, the proven effectiveness of pharmacological interventions (i.e. systemic steroids) is scanty.

6) Bos et al. [8] point out the discrepancy between the favourable results of experimental studies testing interventional treatments on lung animal models and the disappointing results of several clinical randomised controlled trials on ventilatory and pharmacological treatments in ARDS patients. The differences between animal models and human lungs with ARDS, the biological heterogeneity of the disease consequent to the clinical-physiological ARDS definition, and the unselected applications of interventional strategies to the different ARDS "phenotypes" may explain this bias [12]. Within this context, precision medicine is required to find the best balance between harm and effectiveness in the selected patterns of disease. Computer algorithms may be of help in translating complex datasets of different variables in distinct ARDS "phenotypes" that are likely to benefit from different therapeutic ventilatory and non-ventilatory strategies. For example, it seems that the "inflammatory" phenotype is likely to respond to higher PEEP and conservative fluid management while the "hypo-inflammatory" phenotype could be harmed by this intervention [13].

What could we add to the insights of this review that may be of further help for physicians both in clinical practice and the research field? Self-inflicted lung injury is a new concept that has to be born in mind when facing severely hypoxaemic unsupported patients. Given the fact that distressed-induced hyperventilation is likely to increase lung damage in spontaneously breathing ARDS patients, the application of protective IMV could be less harmful and may prevent development of ventilator-induced lung injury [14]. This hypothesis could also fit the scenario of NIV-related hyperventilatory pattern in severely hypoxaemic patients as was suggested by the correlation found between tidal volume values $>9.5 \mathrm{~mL} \cdot \mathrm{kg}^{-1}$ predicted body weight and mortality rate in noninvasively ventilated ARDS [11]. Could high-flow nasal therapy be a less harmful alternative? The report of a better outcome being achieved with high-nasal flow therapy compared to NIV in ARDS seems to support this hypothesis [5]. Physiological studies are required to demonstrate the occurrence of NIV-induced lung damage according to the degree of volume support; furthermore, the benefit of an early application of a high-nasal flow therapy correlated protective noninvasive strategy should be clearly assessed, especially in comparison with NIV in the milder degree of ARDS.

Another important issue is the "real life" management of ARDS in terms of physician's adherence to the recommended "protective ventilatory strategies" and of variability in the recognition and treatment in different geographic regions. According to the findings of a recent observational study performed in $>3000$ ARDS patients managed in 50 ICUs around the world, this syndrome appeared to be under recognised and undertreated and associated with a high mortality rate [3]. Clinical recognition of ARDS ranged from $51.3 \%$ in mild to $78.5 \%$ in severe ARDS. Less than two-thirds of patients with ARDS received protective lung ventilation; plateau pressure was measured in only $40.1 \%$ of the cases whereas $<20 \%$ of patients received PEEP levels $>12 \mathrm{cmH} 2 \mathrm{O}$. Prone positioning was used in $16.3 \%$ of patients with severe ARDS. Conversely, clinician recognition of ARDS was associated with higher PEEP, greater use of neuromuscular blockade and prone positioning. Moreover, important geo-economic differences in the severity, clinician recognition and management of ARDS, and in patients' outcomes have been reported recently [15]. Very disappointingly, clinical outcomes of ARDS are independently associated with economic resources; in fact, hospital survival was significantly lower in middle-income countries than in high-income countries.

To summarise, the European Respiratory Review presents a timely overview of the monitoring and treatment of patients with ARF. This series will help us to optimise diagnosis and treatment for our vulnerable patients and make us realise that there are many unanswered questions that need to be addressed in the near future.

Conflict of interest: L. Heunks reports research grants from Liberate Medical and Orion Pharma, and fees (speaker and travel) from Getinge, outside the submitted work.

References

1 Pisani L, Corcione N, Nava S. Management of acute hypercapnic respiratory failure. Curr Opin Crit Care 2016; 22: 45-52.

2 Matkovic Z, Huerta A, Soler N, et al. Predictors of adverse outcome in patients hospitalised for exacerbation of chronic obstructive pulmonary disease. Respiration 2012; 84: 17-26.

3 Bellani G, Laffey JG, Pham T, et al. Epidemiology, patterns of care, and mortality for patients with acute respiratory distress syndrome in intensive care units in 50 countries. JAMA 2016; 315: 788-800. 
4 Narendra DK, Hess DR, Sessler CN, et al. Update in management of severe hypoxemic respiratory failure. Chest 2017; 152: 867-879.

5 Frat JP, Coudroy R, Marjanovic N, et al. High-flow nasal oxygen therapy and noninvasive ventilation in the management of acute hypoxemic respiratory failure. Ann Transl Med 2017; 5: 297.

6 Scala R. Challenges on non-invasive ventilation to treat acute respiratory failure in the elderly. BMC Pulm Med 2016; 16: 150.

7 Morelli A, Del Sorbo L, Pesenti A, et al. Extracorporeal carbon dioxide removal $\left(\mathrm{ECCO}_{2} \mathrm{R}\right)$ in patients with acute respiratory failure. Intensive Care Med 2017; 43: 519-530.

8 Bos LD, Martin-Loeches I, Schultz MJ. ARDS: challenges in patient care and frontiers in research. Eur Respir Rev 2018; 27: 170107.

9 Chiumello D, Brochard L, Marini JJ, et al. Respiratory support in patients with acute respiratory distress syndrome: an expert opinion. Crit Care 2017; 21: 240.

10 Rochwerg B, Brochard L, Elliott MW, et al. Official ERS/ATS clinical practice guidelines: noninvasive ventilation for acute respiratory failure. Eur Respir J 2017; 50: 1602426.

11 Carteaux G, Millán-Guilarte T, De Prost N, et al. Failure of noninvasive ventilation for de novo acute hypoxemic respiratory failure. Crit Care Med 2016; 44: 282-290.

12 Calfee CS, Delucchi K, Parsons PE, et al. Subphenotypes in acute respiratory distress syndrome: latent class analysis of data from two randomised controlled trials. Lancet Respir Med 2014; 2: 611-620.

13 Bos LD, Schouten LR, van Vught LA, et al. Identification and validation of distinct biological phenotypes in patients with acute respiratory distress syndrome by cluster analysis. Thorax 2017; 72: 876-883.

14 Brochard L, Slutsky A, Pesenti A. Mechanical ventilation to minimize progression of lung injury in acute respiratory failure. Am J Respir Crit Care Med 2017; 195: 438-442.

15 Laffey JG, Madotto F, Bellani G, et al. Geo-economic variations in epidemiology, patterns of care, and outcomes in patients with acute respiratory distress syndrome: insights from the LUNG SAFE prospective cohort study. Lancet Respir Med 2017; 5: 627-638. 